\title{
Structures of SdrD from Staphylococcus aureus reveal the molecular mechanism of how the cell surface receptors recognize their ligands
}

\author{
Xiao Wang ${ }^{1}$, Jingpeng $\mathrm{Ge}^{1}$, Bao $\mathrm{Liu}^{2}$, Yulin $\mathrm{Hu}^{3}$, Maojun Yang ${ }^{1 凶}$ \\ ${ }^{1}$ Key Laboratory for Protein Sciences of Ministry of Education, Tsinghua-Peking Center for Life Sciences, School of Life Sciences, \\ Tsinghua University, Beijing 100084, China \\ 2 Department of Vascular Surgery, Peking Union Medical College Hospital, Beijing 100005, China \\ ${ }^{3}$ Division of Hepatobiliary-Pancreatic, The First Hospital of JiLlin University, Changchun 130021, China \\ $\triangle$ Correspondence: maojunyang@tsinghua.edu.cn \\ Received January 23, 2013 Accepted February 25, 2013
}

\begin{abstract}
Staphylococcus aureus is the most important Gram-positive colonizer of human skin and nasal passage, causing high morbidity and mortality. SD-repeat containing protein D (SdrD), an MSCRAMM (Microbial Surface Components Recognizing Adhesive Matrix Molecules) family surface protein, plays an important role in $S$. aureus adhesion and pathogenesis, while its binding target and molecular mechanism remain largely unknown. Here we solved the crystal structures of SdrD N2-N3 domain and N2-N3-B1 domain. Through structural analysis and comparisons, we characterized the ligand binding site of SdrD, and proposed a featured sequence motif of its potential ligands. In addition, the structures revealed for the first time the interactions between B1 domain and N2-N3 domain among B domain-containing MSCRAMMs. Our results may help in understanding the roles SdrD plays in S. aureus adhesion and shed light on the development of novel antibiotics.
\end{abstract}

KEYWORDS SdrD, adhesin, MSCRAMM, Staphylococcus aureus

\section{INTRODUCTION}

Staphylococcus aureus (S. aureus), widely distributed around the world (Kluytmans et al., 1997), is a major threat to humans leading to infections ranging from mild skin infections to severe syndromes including meningitis, pneumonia, endocarditis, bacteremia, osteomyelitis and sepsis (Mongkolrattanothai et al., 2003). Due to the increasing cases of antibiotic-resistant S. aureus (Diekema et al., 2001; Lowy, 2003; Gordon and Lowy, 2008), S. aureus has raised more concern and public anxiety, further arousing the requirement of new antibiotics (Mongkolrattanothai et al., 2003; Moran et al., 2006; Schito, 2006). S.aureus pathogenesis involves a complex system of cell surface-associated proteins (adhesins) and extracellular toxins (Weber, 2005; Maltezou and Giamarellou, 2006). The presence of the adhesins, named Microbial Surface Components Recognizing Adhesive Matrix Molecules (MSCRAMMs) (Foster and Hook, 1998), enables S. aureus to survive on the surface of a variety of host tissues (Mongkolrattanothai et al., 2003). MSCRAMMs recognize and adhere to the extracellular matrix proteins (Patti and Hook, 1994), and then initialize the pathogenesis process (Costerton et al., 1999). SD-repeat containing (Sdr) proteins constitute a subfamily of the MSCRAMM family (McCrea et al., 2000), including members such as clumping factor A (ClfA), ClfB, SdrC, SdrD and SdrE of S. aureus and SdrF and SdrG of $S$. epidermidis. This protein family is characterized by the unique structural element- $R$ region mainly composed of repeating Ser-Asp dipeptides.

The Sdr family members share a conserved structural organization (Fig. 1A) (Trad et al., 2004). They all start with a short $\mathrm{N}$-terminal signal sequence followed by an $\mathrm{A}$ region for ligand binding. The characteristic $\mathrm{R}$ region differs in length, and is further followed by an LPXTG cell wall-anchoring motif (W), a hydrophobic membrane region (M), and sometimes a cytoplasmic tail (C) (Downer et al., 2002). The ligand-interacting $A$ region is further divided into distinct functional sub-domains, named N1, N2 and N3 domain. Only $20 \%-30 \%$ of amino acid 


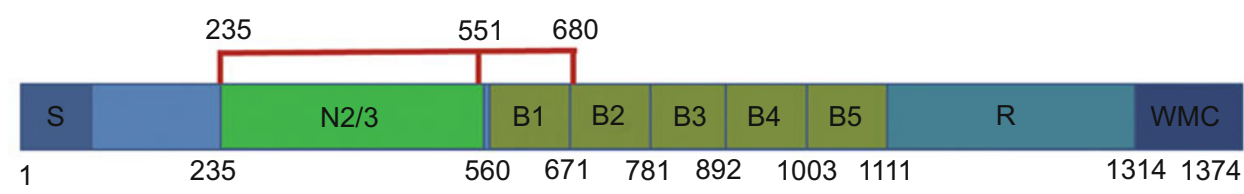

B

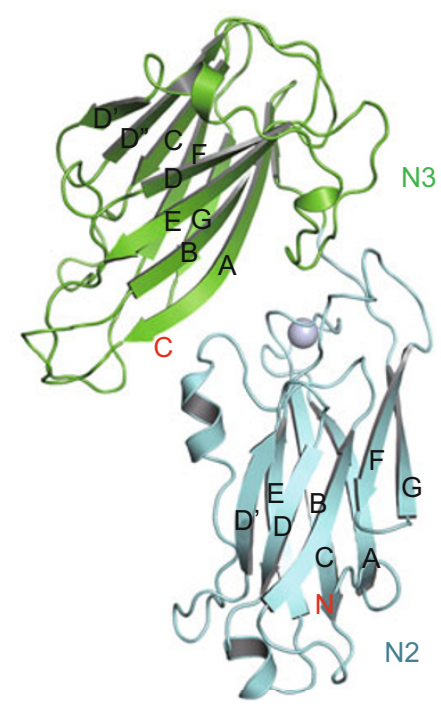

C

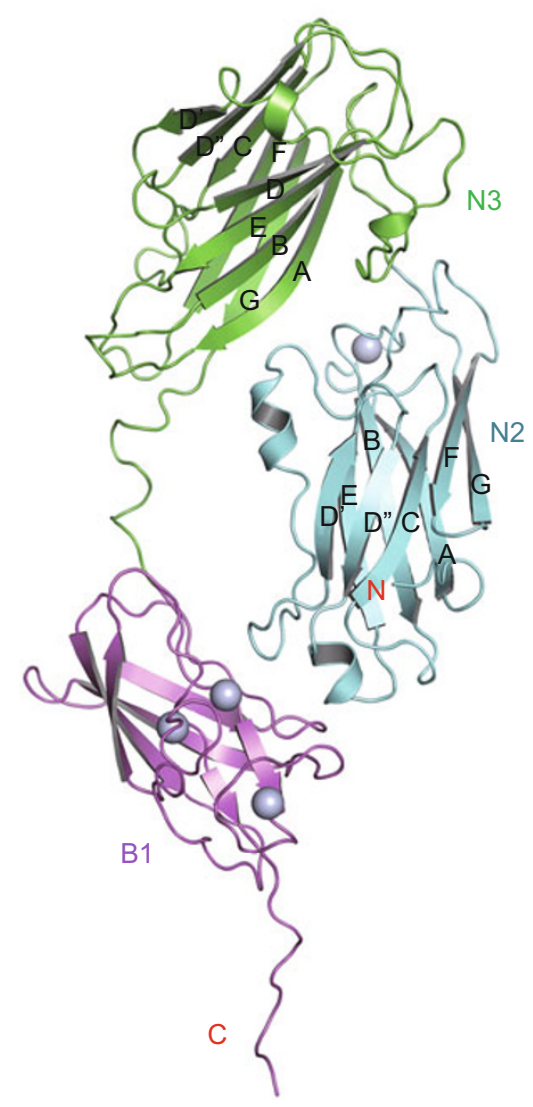

Figure 1. Structural organization and overall view of SdrD. (A) The domain structure of SdrD. S, the N-terminal signal sequence; N2/N3, ligand-binding subdomains; B, five B-repeats; R, SD-repeating domain; WMC, Wall-spanning segment, Membrane-spanning segment and cytoplasmic C-terminal end. The amino acid residue number identifying the boundary between each subdomain is indicated below, and the boundary of the recombinant segments is indicated above. (B) Cartoon representation of the structure of $\mathrm{SdrD}_{(235-551) \text {. }}$ (C) Cartoon representation of the structure of $\mathrm{SdrD}_{(235-680)}$. Domain N2, N3 and B1 are colored in cyan, green and magenta, respectively. Calcium ions are shown as lightblue spheres. The $\mathrm{N}$ and $\mathrm{C}$ termini and strand numbers are designated.

residues are identical among the $\mathrm{N}$ domains from different proteins in this family, suggesting that various roles might be played by different proteins in the ligand binding process. Apart from these basic structural elements, SdrC, SdrE and SdrD all have several (two, three and five, respectively) additional $B$ domains inserted between region $A$ and $R$. The $B$ domain, composed of 110-113 amino acid residues, often exists as a series of repeats (Fig. 1A). Due to the different numbers of repeats in different Sdr proteins, B domains may act as a spacer, finetuning the distance between the ligand-binding $A$ region and $S$. aureus cell surface, thus endowing $S$. aureus with the ability of flexibly adhering to multiple hosts. B domains share significant sequence similarities, including a sequence corresponding to EF-hand motif with an identity of over $35 \%$, and two conserved $\mathrm{Ca}^{2+}$ binding sites with $32 \%$ and $20 \%$ identity, respectively (Josefsson et al., 1998).

Previous studies into the crystal structures of ClfA, ClfB and SdrG have revealed the molecular mechanism underlying ligand-binding by $A$ region in these MSCRAMMs (Ponnuraj et al., 2003; Ganesh et al., 2008). Typically, MSCRAMMs have been reported to bind to fibrinogen, fibronectin, neurexin and IgGs (Hartford et al., 2001; Deivanayagam et al., 2002; Barbu et al., 2010; Ganesh et al., 2011). Our recent work has also revealed a multi-ligand binding mechanism of ClfB, thus raising the possibility that multi-ligand binding might be a common characteristic shared by multiple MSCRAMMs (Xiang et al., 2012). However, the biological functions and binding targets of SdrD were not understood. Furthermore, the functions of $B$ domains in SdrC, SdrE and SdrD also remain elusive (Foster and Hook, 1998; Josefsson et al., 1998). To address these, we solved the crystal structures of the ligand-binding sub-domain N2-N3 and ligand-binding-spacer region N2-N3-B1 of SdrD. Our structures revealed a similar ligand binding region in SdrD as those in the established structures such as ClfB. The interactions between N2-N3 domain and B domain indicated the spacer role of $B$ region and revealed how the ligands binding $A$ region was projected to the extracellular region. These results would be valuable for identifying the potential ligand of SdrD, 
and shed light upon the development of novel and different therapeutic strategies.

\section{RESULTS}

\section{Structures of N2-N3 and N2-N3-B1 domain of SdrD}

Previous studies into ClfB and SdrG have demonstrated that the ligand-binding region was located within the N2 and N3 domains (Ganesh et al., 2011; Ponnuraj et al., 2003). Based on sequence alignment, we cloned a segment of SdrD from $S$. aureus (residues 235-551) corresponding to both N2 and N3 domains and solved its crystal structure. The structure of SdrD N2-N3 consists of residues Lys235-GIn551 (Fig. 1B). As anticipated, the polypeptide in the structure folds into two domains, $\mathrm{N} 2$ and N3, with a loose loop linking them together. The overall folds of N2 and N3 both follow a DE variant lgG model (Fig. 1B) (Deivanayagam et al., 2002). Each one is made of two layers of $\beta$ sheets packed against each other, eight in N2, and ten in N3. In N2 domain, strands C, D', D", F and G form one principle layer, and strands $A, B$ and $E$ constitute the other one. The N3 domain folds in largely the same conformation as N2, with a root mean square deviation (RMSD) of $0.60 \AA$ over 99 $C \alpha$ atoms, except that an additional strand $D$ is formed in the $A, B$ and $E$ layer and another strand $A^{\prime}$ is in the opposite layer. In N3, two a helices exist in the same location as those in N2 although in a different topological order. $\mathrm{A} \mathrm{Ca}^{2+}$ ion is located within the loop region of $\mathrm{N} 2$ domain, on the interface between the N2 and N3 domains. Typically, the overall structure of N2N3 domain is consistent with the former structures of ClfA, ClfB and SdrG, indicating their similarities in working mechanisms.

To elucidate the functions of B domain in Sdr family proteins, we further cloned a segment of SdrD (residues 235-680) corresponding to N2-N3-B1 region (Fig. 1A) and solved its crystal structure. The structure of N2-N3-B1 contains residues from Lys235 to Asp680 (Fig. 1C). The existence of B1 domain does not cause an obvious conformational change in N2 and N3 domain, with an RMSD of $0.39 \AA$ over 273 Ca atoms, between the two structures. B1 domain consists of a barrel of six $\beta$ strands, spanning from residue Val559 to Pro670. B1 is directed away from N3 domain by its N-terminal loop and interacts with N2 domain from the opposite side. C-terminal to the main body of B1 is a loop which directs this domain away from the transmembrane region. (All structural figures in this paper were generated by PyMOL).

\section{Structural comparison of SdrD B1 domain with Cna}

B domains are highly conserved repeats, whose conserved residues cluster at one high affinity $\mathrm{Ca}^{2+}$ binding site and two low affinity binding sites (Fig. 2A). These conserved residues are essential for $\mathrm{Ca}^{2+}$ binding as single mutations ruined the affinity. Consistently, in the structure, three $\mathrm{Ca}^{2+}$ binding sites are identified on the interface between domain B1 and N2 (Fig. 2B), which we named S1, S2 and S3 in accordance with the sequence order of the residues. In detail, $\mathrm{Ca}^{2+}$ ion at $\mathrm{S} 1$ forms more polar contacts with the protein, compared with the two at S2 and S3, possibly explaining the higher binding affinity (Fig. 2B). The binding site $\mathrm{S} 1$ is structurally like an EF-hand motif and the $\mathrm{Ca}^{2+}$ ion is well coordinated by the surrounding residues (Josefsson et al., 1998). While in S2 and S3, the $\mathrm{Ca}^{2+}$ ions are exposed to some extent. Importantly, $\mathrm{Ca}^{2+}$ ions were essential for protein stability during the purification and crystallization processes in our experiment.

Cna from $S$. aureus is an MSCRAMM protein binding to collagen and adapts a similar overall structure pattern with SdrD (Deivanayagam et al., 2000), with a non-repetitive A region, followed by several repetitive $B$ regions. Though the identity between the $B$ domains of the two proteins is low in the amino acid sequence level, structural comparison reveals a spatial resemblance in the overall structure (Fig. 2C). B1 and B2 regions of Cna are packed side by side, and share highly similar folds to each other. Considering the structural similarities between the $B$ domains of the two proteins, we propose that the $B$ domain repeats of SdrD might adapt a similar overall structure.

\section{Structural insights into N2-B1 interaction surface}

In the interface between $\mathrm{N} 2$ and $\mathrm{B} 1, \mathrm{Ca}^{2+}$ binding site $\mathrm{S} 3$ plays an important role (Fig. $3 \mathrm{~A}$ ). $\mathrm{Ca}^{2+}$ ion at $\mathrm{S} 3$ mediates the hydrogen bonds between the two domains. The $\mathrm{N}$-terminal loop and the loop between strand C and D of B1 together form a positively charged calcium binding pocket. This pocket interacts with $\alpha 2$ and the loop between $\alpha 1$ and $\beta$ sheet D' of N2 domain. Several hydrogen bonds are formed and stabilize the interaction between B1 and N2, involving Asn565, Lys608, Leu612 and Leu663 from B1 and Asn313, Glu315, Tyr336 and Arg339 from N2. The interaction between N2 and B1 only causes a slight influence in the structure of N2-N3. For example, the distance between Asp301 and Thr402 extends from $7.2 \AA$ in N2-N3 structure to $7.7 \AA$ in N2-N3-B1 structure (Fig. 3B). This indicates that the binding between these two domains is very weak, which is consistent with the previous "dock, lock, and latch" (DLL) model. Previous studies have indicated that the binding of the ligands to the groove between N2 and N3 would induce the linker between N3 and B1 to form an extra G' strand to lock and latch the ligands. The Gly-rich sequence in this linker is consistent with this proposal. So we propose that the B domains in the SdrD protein might work as the scaffold to protrude the A domain out to bind to the ligands.

\section{Structural comparisons of SdrD N2-N3 domain with ClfB}

Structural alignment of SdrD with other MSCRAMMs with known structures (data not shown) reveals that the structure of N2-N3 of SdrD highly resembles that of ClfB (Fig. 4A). Interestingly, RMSD between SdrD and ClfB-Fga is $2.1 \AA$ over $230 \mathrm{C} \alpha$ atoms, compared with $2.9 \AA$ between $\mathrm{SdrD}$ and apo-ClfB. In order to gain insights into the potential ligands of SdrD, we compared the ligand binding sites of the two structures in detail. Consequently, several conserved residues between SdrD and ClfB are located at the ligand binding site 
A

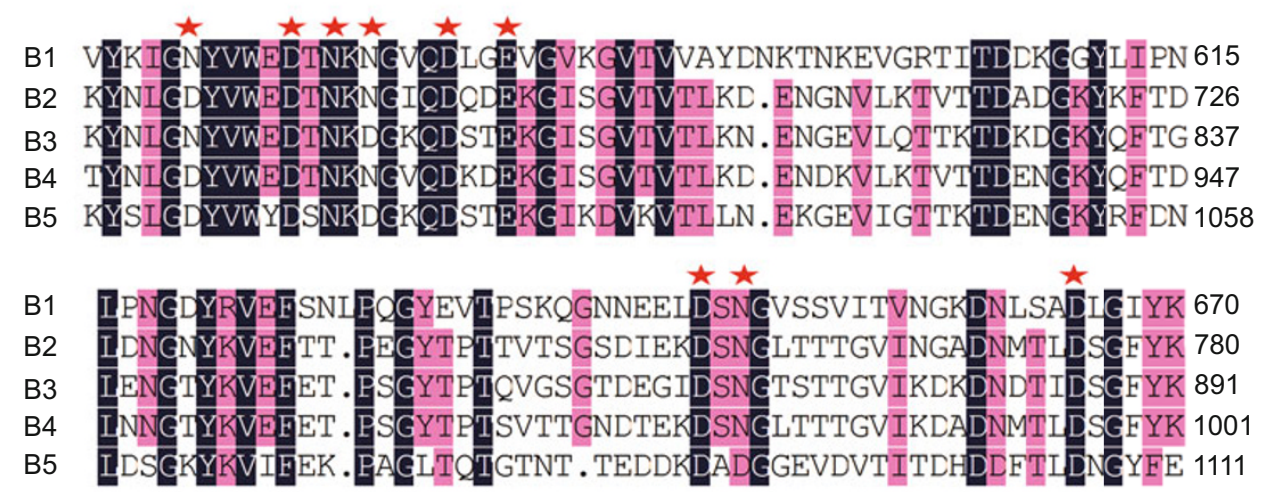

B

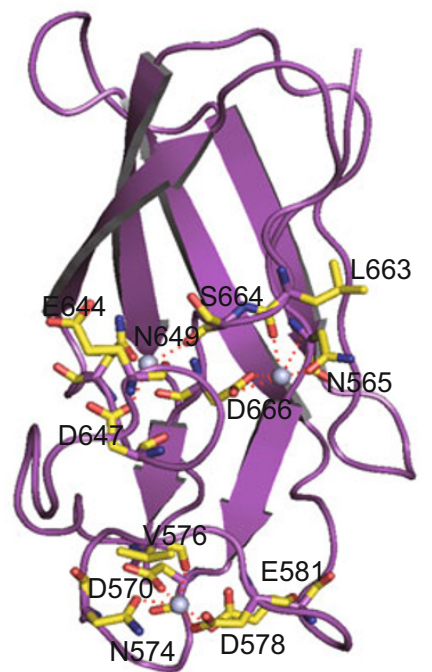

C

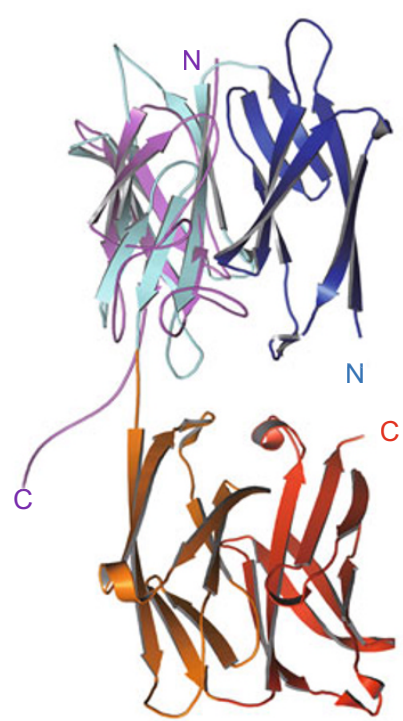

Figure 2. Sequence alignment and comparison with Cna. (A) Sequence alignment of SdrD B domains (amino acid residues 5601111). Residues with $100 \%$ homology and over $75 \%$ homology are shaded in dark blue and pink, respectively. Key residues for calcium binding are marked with stars. (B) Cartoon representation of domain B1. Calcium ions are represented by light blue spheres. Residues interacting with the calcium ions are shown as sticks. Hydrogen bonds are represented as red dashed lines. (C) Superimposition of SdrD B1 domain and Cna. The four repetitive B regions of Cna are colored blue, cyan, orange, and red, respectively. SdrD B1 domain is shown in magenta. The $\mathrm{N}$ and $\mathrm{C}$ termini for both molecules are designated.

(Fig. 4A). Notably, the residues GIn235, Ser236, Phe328, Thr383 and Asn524 are five key residues in mediating the interactions between ClfB and Fga. While in SdrD, the five residues at the corresponding positions show shift within $1 \AA$ between Ca atoms, which are Gln29, Leu63, Tyr117, Glu66 and Asn309, respectively. This indicates that there might be some common structural features shared by the ligands of the two MSCRAMMs.

\section{DISCUSSION}

The detailed molecular mechanism of how the cell surface receptor-SdrD bind its ligand in $S$. aureus pathogenesis remains unknown (Tung et al., 2000). Trying to answer this question, we solved the high resolution crystal structures of SdrD N2-
N3 domain and N2-N3-B1 domain. Structural analysis identified the possible ligand binding groove in the structure of N2N3 domain. Furthermore, the structure of N2-N3-B1 domain revealed a slight conformational change compared with that of the N2-N3 domain. In the structure, B1 domain interacts with $\mathrm{N} 2$ domain, further opening the groove between N2 and N3 for potential ligands to fit in.

The identification of the conserved ligand binding groove between SdrD and ClfB provides important insights into the features of the potential ligands that SdrD binds to (Hartford et al., 2001; Ponnuraj et al., 2003; Ganesh et al., 2011; Xiang et al., 2012). The conserved residues in ClfB mentioned above have been reported to specifically recognize a GSR motif derivative of the ligands (Xiang et al., 2012), with the sequence GSSGXGXXG. Based on the ClfB ligands sequence and 


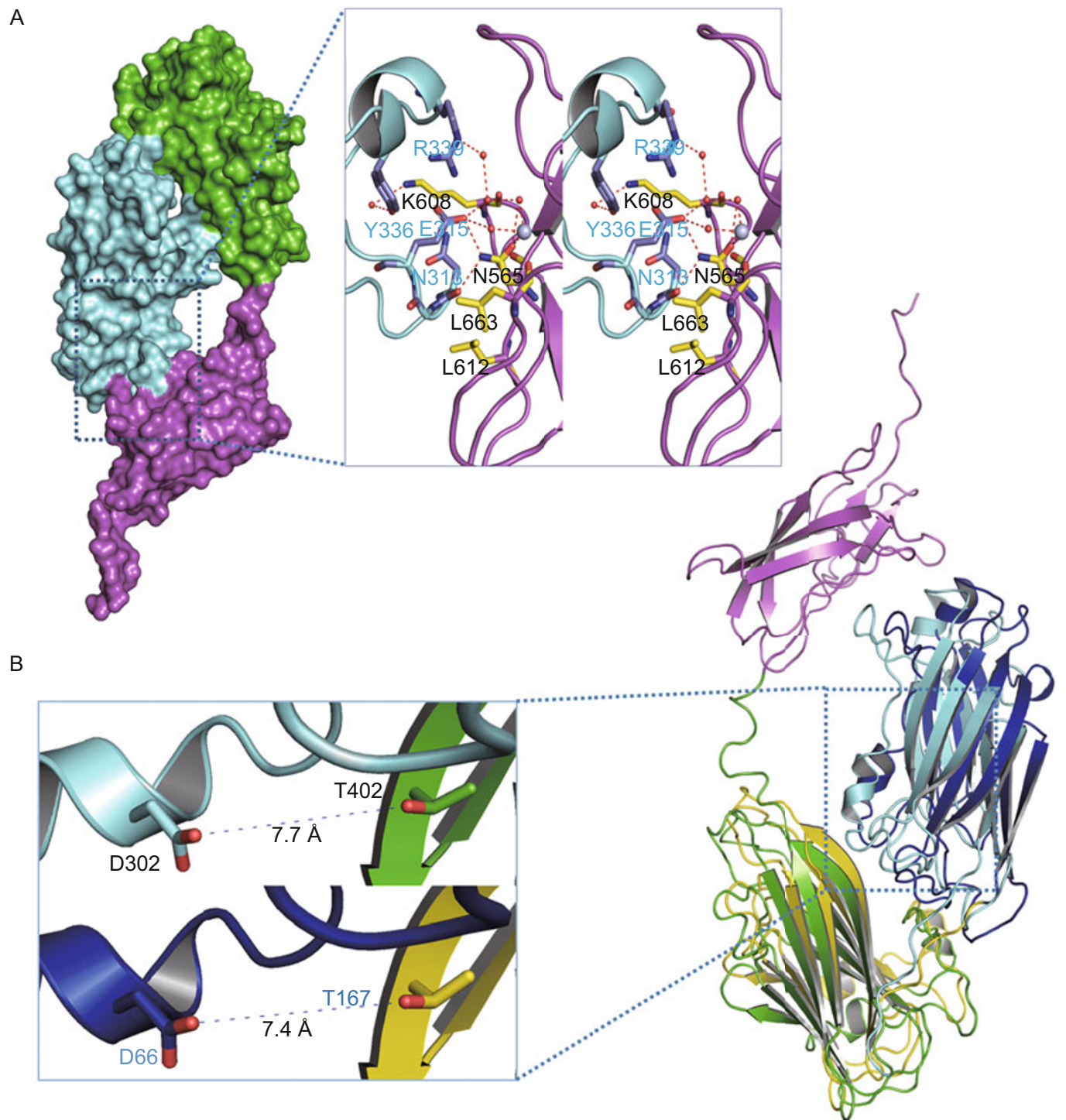

Figure 3. Details of the interaction surface. (A) Interactions between N2 and B1 domain. SdrD is shown in the surface model. The color schemes are the same as in Fig. 1C. The right panel is an amplified view of the region in the box in the left panel. Residues interacting with calcium ions are shown as yellow sticks (B1) and blue sticks (N2). Water molecules and calcium ions are shown as red and lightblue spheres, respectively. Hydrogen bonds are shown as red dashed lines. (B) Superimposition of N2-N3 and N2-N3-B1 domains. The structure of N2-N3-B1 domain is colored the same as in Fig. 1C. For the structure of N2-N3, domain N2 is in blue and N3 in yellow. Asp301 and Thr402 are marked and shown in sticks in the closer view.

the conservation of the ligands binding residues in ClfB and SdrD, we could propose some features of the SdrD ligands. Particularly, the ligands of SdrD should contain a conserved SXGXXXT sequence in the centre. In all, structural comparison studies using SdrD with other proteins with identified ligands might provide important insights into identification of the binding ligand of SdrD.

In addition, based on the $\mathrm{B}$ domain structure of Cna, a model of the whole SdrD protein could be proposed (Fig. 4B): While $\mathrm{W}$ domain is anchored into the cell wall, $\mathrm{R}$ domain serves as a bridge to extend the preceding domains into extracellular space. Moreover, $\mathrm{B}$ domains act as a spring to regulate the location and orientation of ligand binding domains. Finally, N1, $\mathrm{N} 2$ and N3 together constitute the A domain, ready to bind to target ligand and help the $S$. aureus adhere onto different surfaces. This model may be applied to other B domain-containing Sdr proteins, i.e. SdrC and SdrE. Emerging of this new domain may be due to the limited length and orientation of $\mathrm{R}$ domain. The new stretching mechanism contributed by B domain could endow $S$. aureus with abilities to interact with different extracellular components separately and simultaneously.

$S$. aureus pathogenesis remains an important and complex 


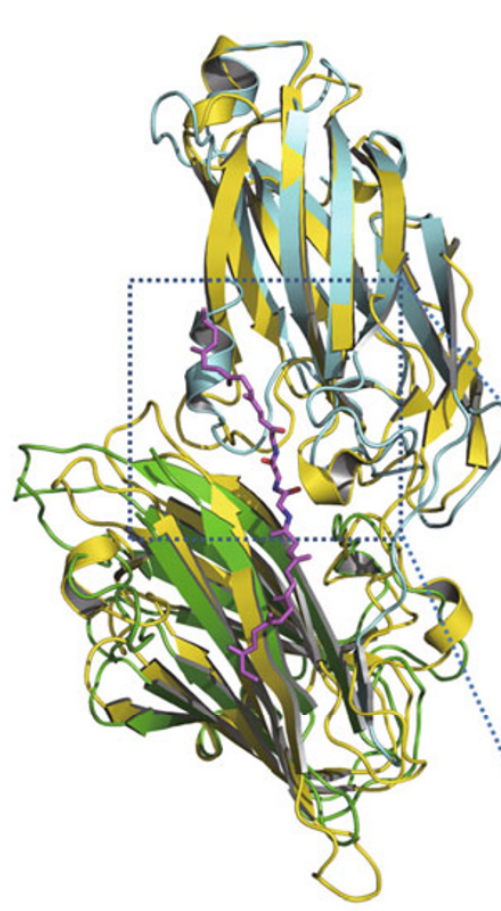

B

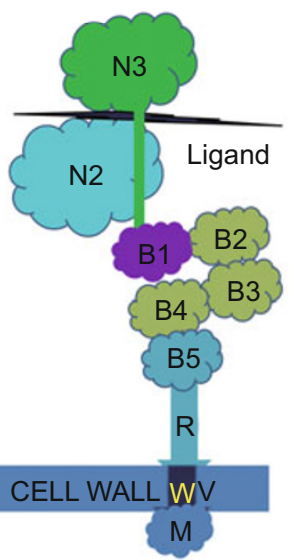

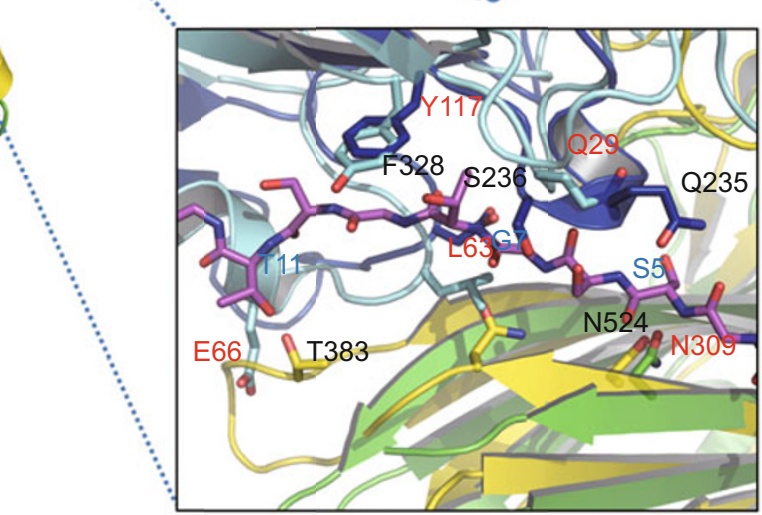

Figure 4. Proposed binding sites and model. (A) Superimposition of SdrD N2-N3 and ClfB-Fga. SdrD is colored the same as in Fig. 1. ClfB protein is colored yellow and the ligand Fga in magenta. Close view outlined the ligand binding region. Side chains are shown as sticks. Residues are labeled in red for SdrD, in black for ClfB and in orange for the ligand. (B) A working model of the SdrD protein. The molecule is stretching from the cell wall, which is indicated at the bottom. The color schemes are the same as in Fig. 1A.

issue, and far from fully understood. Instead of attempting various traditional antibiotics, blocking or modifying $S$. aureus adhering ability could interfere with its survival and invasion. MSCRAMMs are the direct executioners in the adhering process. Structural studies into proteins of this family not only help in understanding its mechanism, but also lead to potential therapy methods (O'Riordan and Lee, 2004). SdrD, one of the key molecules in this process, has been identified to have an increased expression level in bone-related infections (Ponnuraj et al., 2003). Our studies have provided important insights into its working mechanism and the potential ligands, although the details of the mechanism and ligand information still need further investigations. In all, structural characterization of SdrD could significantly promote studies into $S$. aureus pathogenesis and shed light on the development of new antibiotics.

\section{MATERIALS AND METHODS}

Cloning, expression and purification of the recombinant protein

The cDNA sequences corresponding to residues 235-551 and 235-680 of SdrD were amplified and cloned into pGEX6p-1 vector, respectively. The recombinant protein of $\mathrm{SdrD}_{235-680}$ was expressed with a GST fusion tag in the prokaryotic BL21 strain of $E$. coli with high yield. The bacteria were collected and resuspended in lysis buffer ( $1 \times$ PBS, $1 \mathrm{mmol} / \mathrm{L}$ PMSF, and $2 \mathrm{mmol} / \mathrm{L}$ DTT). The cells then were homogenized by sonification on ice. Cell debris was removed completely by centrifuging the lysate at $4^{\circ} \mathrm{C}$ at $13,000 \mathrm{~g}$ for $60 \mathrm{~min}$.

All the following steps were performed at $4^{\circ} \mathrm{C}$. The supernatant was applied onto self-packaged GST-affinity columns $(1.5-2 \mathrm{~mL}$ glutathione Sepharose $4 \mathrm{~B}$ beads) and the contaminant was washed away by lysis buffer. The fusion protein was then digested with PreScission protease at $4^{\circ} \mathrm{C}$ overnight. Then protein with digested remaining five amino acid residues (GPLGS) at the N-terminal was eluted with lysis buffer. Eluant was applied onto Sephadex G-25 gel filtration column in Tris buffer $(25 \mathrm{mmol} / \mathrm{L}$ Tris-HCl pH 8.0, $5 \mathrm{mmol} / \mathrm{L}$ DTT) then onto Resource Q (Pharmacia) column (buffer A: 25 mmol/L Tris- HCl pH 8.0, $5 \mathrm{mmol} / \mathrm{L}$ DTT; buffer B: $25 \mathrm{mmol} / \mathrm{L}$ Tris- $\mathrm{HCl} \mathrm{pH}$ 8.0, $500 \mathrm{mmol} / \mathrm{L} \mathrm{NaCl}$, $5 \mathrm{mmol} / \mathrm{L}$ DTT). Pooled fractions containing the target protein were gathered and concentrated using an Ultrafree 10,000 molecularweight cutoff filter unit (Millipore) and further purified using a Superdex-200 (Pharmacia) column (Try buffer: $10 \mathrm{mmol} / \mathrm{L}$ Tris-HCl, $\mathrm{pH}$ 8.0, $100 \mathrm{mmol} / \mathrm{L} \mathrm{NaCl}, 2 \mathrm{mmol} / \mathrm{L} \mathrm{CaCl}$, $1 \mathrm{mmol} / \mathrm{L} \mathrm{DTT})$. The selenomethionine (Se-Met) protein of $\mathrm{SdrD}_{235-551}$ was expressed and purified similarly. Protein in each step was analyzed by SDS-PAGE Coomassie Brilliant Blue staining (Zhang et al., 2010). 
Table 1. Statistics of data collection and refinement

\begin{tabular}{|c|c|c|}
\hline Peptide & $\begin{array}{l}\text { SdrD N2-N3 } \\
\text { Se-SAD }\end{array}$ & $\begin{array}{l}\text { SdrD N2-N3-B1 } \\
\text { Native }\end{array}$ \\
\hline \multicolumn{3}{|l|}{ Data collection } \\
\hline Wavelength $(\AA)$ & 0.979 & 0.979 \\
\hline Space Group & $\mathrm{C} 22_{1}$ & $P 2_{1}$ \\
\hline Unit Cell $(\AA)$ & $\begin{array}{l}133.31,58.36,112.36 \\
\alpha=90.00, \beta=111.14, \gamma=90.00\end{array}$ & $\begin{array}{l}74.41,73.88,90.03 \\
\alpha=90.00, \beta=102.42, y=90.00\end{array}$ \\
\hline Resolution $(\AA)$ & $1.70(1.76-1.70)$ & $2.10(2.14-2.10)$ \\
\hline$R_{\text {merge }}(\%)$ & $6.1(46.0)$ & $6.2(29.6)$ \\
\hline //sigma & $13.1(2.4)$ & $33.0(4.3)$ \\
\hline Completeness (\%) & $96.6(95.3)$ & $99.4(92.0)$ \\
\hline Redundancy & $3.8(2.0)$ & $4.1(3.6)$ \\
\hline No. reflections & 85801 & 55836 \\
\hline Wilson B factor $\left(\AA^{2}\right)$ & 28.6 & 34.0 \\
\hline \multicolumn{3}{|l|}{ SAD phasing } \\
\hline Anomalous caterers & $8 \mathrm{Se}$ & \\
\hline Figure-of-merit (FOM) & 0.397 & \\
\hline FOM after DM & 0.674 & \\
\hline FOM after phase combination & 0.778 & \\
\hline \multicolumn{3}{|l|}{ Refinement } \\
\hline$R$ factor & 0.2085 & 0.1847 \\
\hline$R_{\text {free }}$ & 0.2462 & 0.2315 \\
\hline No. atoms & 4852 protein atoms $+1 \mathrm{Ca}^{2+}$ & 7250 protein atoms $+8 \mathrm{Ca}^{2+}$ \\
\hline \multicolumn{3}{|l|}{ B factors } \\
\hline Overall & 45.9 & 53.4 \\
\hline Main chain & 42.6 & 51.3 \\
\hline Side chain & 47.8 & 57.1 \\
\hline Macromolecules & 45.1 & 54.1 \\
\hline Ligands & 51.5 & 36.0 \\
\hline Solvent & 52.1 & 43.3 \\
\hline \multicolumn{3}{|l|}{ Ramachandran plot statistics (\%) } \\
\hline In preferred regions & 98.0 & 93.6 \\
\hline In allowed regions & 2.0 & 4.9 \\
\hline Outliers & 0.0 & 1.5 \\
\hline
\end{tabular}

Values in parentheses are for the highest resolution shell. $R=\Sigma \mid F_{\text {obs }}-F_{\text {calc }} / \Sigma F_{\text {obs, }}$ where $F_{\text {calc }}$ is the calculated protein structure factor from the atomic model ( $R_{\text {free }}$ was calculated with $5 \%$ of the reflections).

\section{Crystallization}

The purified protein was concentrated to about $25 \mathrm{mg} / \mathrm{mL}$. Protein concentrations were determined by absorbance at $280 \mathrm{~nm}$, assuming an A280 of 1.046 for a $1.0 \mathrm{mg} / \mathrm{mL}$ solution. The protein sample was centrifuged at $10,000 \mathrm{~g}$ for $10 \mathrm{~min}$ to clarify the solution before initiating any crystal trials. Initial screening was performed at $18^{\circ} \mathrm{C}$ in 24 -well plates by the hanging-drop vapor-diffusion method using the sparse-matrix screen kits from Hampton Research (Crystal Screen reagent kits I and II) (Jancarik et al., 1991). Crystals were obtained and the diffraction was improved by a refinement of the conditions through the variation of pre- cipitants, $\mathrm{pH}$, protein concentrations and additives. Typically, hanging droplets consist of $21 \mathrm{mg} / \mathrm{mL}$ protein solution and an equivalent volume of reservoir. Crystals were grown at $18^{\circ} \mathrm{C}$ by mixing $1.1 \mu \mathrm{L}$ protein with $1.1 \mu \mathrm{L}$ reservoir solution, against $200 \mu \mathrm{L}$ of reservoir solution.

\section{X-ray crystallographic studies}

Native and Se-SAD data were both collected at Shanghai Synchrotron Radiation Facility (SSRF) at a wavelength of $0.979 \AA$ using a MAR225 (MAR Research, Hamburg) CCD detector at $100 \mathrm{~K}$ and processed with HKL2000 (Otwinowski and Minor, 1997). Further processing 
was carried out using programs from the CCP4 suite (Collaborative Computational Project, 1994). The selenium sites were located using SHELXs from the Bijvoet differences in the Se-SAD data (Schneider and Sheldrick, 2002). Heavy atom positions were refined and phases were calculated using PHASER's SAD experimental phasing module (McCoy et al., 2007). The model building of the SdrD molecules was accomplished in COOT and all the structures were refined with the PHENIX packages (Adams et al., 2002; Emsley and Cowtan, 2004). Data collection and structural statistics are summarized in Table 1.

\section{ACCESSION CODES}

Protein Data Bank: The atomic coordinates and structure factors of the SdrD N2-N3 domains and N2-N3-B1 domains have been deposited with accession codes 4JE0, and 4JDZ, respectively.

\section{ACKNOWLEDGMENTS}

We would like to thank the staff at the SSRF BL17U beamline for assistance in data collection. This work was supported by the National Basic Research Program (973 Program) (Nos. 2011CB910502 and 2012CB911101) and National Natural Science Foundation of China (Grant Nos. 31030020 and 31170679).

\section{ABBREVIATIONS}

MSCRAMM, microbial surface components recognizing adhesive matrix molecules; RMSD, root mean square deviation; SdrD, SD-repeat containing protein $\mathrm{D}$

\section{CONFLICT OF INTEREST}

Xiao Wang, Jingpeng Ge, Bao Liu, Yulin Hu, and Maojun Yang declare that they have no conflict of interest.

This article does not contain any studies with human or animal subjects performed by the any of the authors.

\section{REFERENCES}

Adams, P.D., Grosse-Kunstleve, R.W., Hung, L.W., loerger, T.R., McCoy, A.J., Moriarty, N.W., Read, R.J., Sacchettini, J.C., Sauter, N.K., and Terwilliger, T.C. (2002). PHENIX: building new software for automated crystallographic structure determination. Acta Crystallogr. Sect. D-Biol. Crystallogr. 58, 1948-1954.

Barbu, E.M., Ganesh, V.K., Gurusiddappa, S., Mackenzie, R.C., Foster, T.J., Sudhof, T.C., and Hook, M. (2010). beta-Neurexin is a ligand for the Staphylococcus aureus MSCRAMM SdrC. PLoS Pathog. 6, 11.

Costerton, J.W., Stewart, P.S., and Greenberg, E.P. (1999). Bacterial biofilms: A common cause of persistent infections. Science 284, 1318-1322.

Deivanayagam, C.C.S., Rich, R.L., Carson, M., Owens, R.T., Danthuluri, S., Bice, T., Hook, M., and Narayana, S.V.L. (2000). Novel fold and assembly of the repetitive $B$ region of the Staphylococcus aureus collagen-binding surface protein. Struct Fold Des 8, 67-78.

Deivanayagam, C.C.S., Wann, E.R., Chen, W., Carson, M., Rajashankar, K.R., Hook, M., and Narayana, S.V.L. (2002). A novel variant of the immunoglobulin fold in surface adhesins of Staphylococcus aureus: crystal structure of the fibrinogen-binding
MSCRAMM, clumping factor A. EMBO J 21, 6660-6672.

Diekema, D.J., Pfaller, M.A., Schmitz, F.J., Smayevsky, J., Bell, J., Jones, R.N., Beach, M., and Grp, S.P. (2001). Survey of infections due to Staphylococcus species: Frequency of occurrence and antimicrobial susceptibility of isolates collected in the United States, Canada, Latin America, Europe, and the Western Pacific region for the SENTRY Antimicrobial Surveillance Program, 1997-1999. Clin Infect Dis 32, S114-132.

Downer, R., Roche, F., Park, P.W., Mecham, R.P., and Foster, T.J. (2002). The elastin-binding protein of Staphylococcus aureus (EbpS) is expressed at the cell surface as an integral membrane protein and not as a cell wall-associated protein. J Biol Chem 277, 243-250.

Emsley, P., and Cowtan, K. (2004). Coot: model-building tools for molecular graphics. Acta Crystallogr Sect D-Biol Crystallogr 60, 2126-2132.

Foster, T.J., and Hook, M. (1998). Surface protein adhesins of Staphylococcus aureus. Trends Microbiol 6, 484-488.

Ganesh, V.K., Barbu, E.M., Deivanayagam, C.C.S., Le, B., Anderson, A.S., Matsuka, Y.V., Lin, S.L., Foster, T.J., Narayana, S.V.L., and Hook, M. (2011). Structural and biochemical characterization of Staphylococcus aureus clumping factor B/ligand interactions. J Biol Chem 286, 25963-25972.

Ganesh, V.K., Rivera, J.J., Smeds, E., Ko, Y.P., Bowden, M.G., Wann, E.R., Gurusiddappa, S., Fitzgerald, J.R., and Hook, M. (2008). A structural model of the Staphylococcus aureus ClfA-fibrinogen interaction opens new avenues for the design of anti-Staphylococcal therapeutics. PLoS Pathog. 4, 10.

Gordon, R.J., and Lowy, F.D. (2008). Pathogenesis of methicillinresistant Staphylococcus aureus infection. Clin Infect Dis 46, S350-359.

Hartford, O.M., Wann, E.R., Hook, M., and Foster, T.J. (2001). Identification of residues in the Staphylococcus aureus fibrinogen-binding MSCRAMM clumping factor A (ClfA) that are important for ligand binding. J. Biol. Chem. 276, 2466-2473.

Jancarik, J., Scott, W.G., Milligan, D.L., Koshland, D.E., and Kim, S.H. (1991). Crystallization and preliminary-x-ray diffraction study of the ligand-binding domain of the bacterial chemotaxis-mediating aspartate receptor of salmonella-typhimurium. J Mol Biol 221, 31-34.

Josefsson, E., O'Connell, D., Foster, T.J., Durussel, I., and Cox, J.A. (1998). The binding of calcium to the B-repeat segment of SdrD, a cell surface protein of Staphylococcus aureus. J Biol Chem 273, 31145-31152.

Kluytmans, J., vanBelkum, A., and Verbrugh, H. (1997). Nasal carriage of Staphylococcus aureus: Epidemiology, underlying mechanisms, and associated risks. Clin Microbiol Rev 10, 505-520.

Lowy, F.D. (2003). Antimicrobial resistance: the example of Staphylococcus aureus. J Clin Invest 111, 1265-1273.

Maltezou, H.C., and Giamarellou, H. (2006). Community-acquired methicillin-resistant Staphylococcus aureus infections. Int J Antimicrob Agents 27, 87-96.

McCoy, A.J., Grosse-Kunstleve, R.W., Adams, P.D., Winn, M.D., Storoni, L.C., and Read, R.J. (2007). Phaser crystallographic software. J Appl Crystallogr 40, 658-674.

McCrea, K.W., Hartford, O., Davis, S., Eidhin, D.N., Lina, G., Speziale, P., Foster, T.J., and Hook, M. (2000). The serine-aspartate repeat (Sdr) protein family in Staphylococcus epidermidis. Microbiology (UK) 146, 1535-1546. 
Mongkolrattanothai, K., Boyle, S., Kahana, M.D., and Daum, R.S. (2003). Severe Staphylococcus aureus infections caused by clonally related community-acquired methicillin-susceptible and methicillin-resistant isolates. Clin Infect Dis 37, 1050-1058.

Moran, G.J., Krishnadasan, A., Gorwitz, R.J., Fosheim, G.E., McDougal, L.K., Carey, R.B., Talan, D.A., and Grp, E.M.I.N.S. (2006). Methicillin-resistant S-aureus infections among patients in the emergency department. N Engl J Med 355, 666-674.

O'Riordan, K., and Lee, J.C. (2004). Staphylococcus aureus capsular polysaccharides. Clin Microbiol Rev 17, 218-234.

Otwinowski, Z., and Minor, W. (1997). Processing of X-ray diffraction data collected in oscillation mode. Methods Enzymol 276, 307-326.

Patti, J.M., and Hook, M. (1994). Microbial adhesins recognizing extracellular-matrix macromolecules. Curr Opin Cell Biol 6, 752-758.

Ponnuraj, K., Bowden, M.G., Davis, S., Gurusiddappa, S., Moore, D., Choe, D., Xu, Y., Hook, M., and Narayana, S.V.L. (2003). A "dock, lock, and latch" structural model for a staphylococcal adhesin binding to fibrinogen. Cell 115, 217-228.

Schito, G.C. (2006). The importance of the development of antibiotic resistance in Staphylococcus aureus. Clin Microbiol Infect 12, 3-8.
Schneider, T.R., and Sheldrick, G.M. (2002). Substructure solution with SHELXD. Acta Crystallogr Sect D-Biol Crystallogr 58, 1772-1779.

Trad, S., Allignet, J., Frangeul, L., Davi, M., Vergassola, M., Couve, E., Morvan, A., Kechrid, A., Buchrieser, C., Glaser, P., et al. (2004). DNA macroarray for identification and typing of Staphylococcus aureus isolates. J Clin Microbiol 42, 2054-2064.

Tung, H.S., Guss, B., Hellman, U., Persson, L., Rubin, K., and Ryden, C. (2000). A bone sialoprotein-binding protein from Staphylococcus aureus: a member of the staphylococcal Sdr family. Biochem J 345, 611-619.

Weber, J.T. (2005). Community-associated methicillin-resistant Staphylococcus aureus. Clin Infect Dis 41, S269-272.

Xiang, H., Feng, Y., Wang, J.W., Liu, B., Chen, Y.G., Liu, L., Deng, X.M., and Yang, M.J. (2012). Crystal structures reveal the multi-ligand binding mechanism of Staphylococcus aureus ClfB. Plos Pathogens 8, 2751-2751.

Zhang, L.Q., Xiang, H., Gao, J.L., Hu, J., Miao, S.Y., Wang, L.F., Deng, X.M., and Li, S.T. (2010). Purification, characterization, and crystallization of the adhesive domain of SdrD from Staphylococcus aureus. Protein Expr Purif 69, 204-208. 